\title{
ARQUEOLOGÍA DE LOS COLORES. ANÁLISIS MEB-EDX DE MEZCLAS PIGMENTARIAS EN CERÁMICAS PREHISPÁNICAS DE LA CUENCA DEL RÍO PARANÁ (PROVINCIA DE ENTRE RÍOS, NORDESTE DE ARGENTINA)

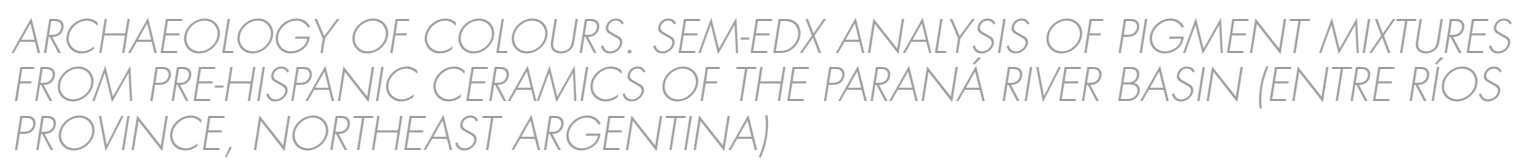

Flavia V. Ottalagano*, Silvia A. DOMÍNGUEZ * * \&ATRICIA B. BOZZANO***

Se presentan los primeros resultados del análisis fisicoquímico de mezclas pigmentarias rojas y blancas empleadas en la decoración de cerámicas arqueológicas provenientes de sitios localizados en la cuenca media y baja del río Paraná (provincia de Entre Ríos, nordeste de Argentina). El material analizado corresponde a conjuntos cerámicos asociados a grupos Goya-Malabrigo, cronológicamente situados al final del Holoceno Tardío. Se utiliza la técnica MEB-EDX a fin de aportar datos relativos a la caracterización elemental de las pinturas, en su fracción inorgánica. La información obtenida señala altas concentraciones de Fe en las pinturas rojas, en tanto que aquellas blancas muestran cierta variabilidad en su composición, destacándose niveles elevados de Ca y P. Estos resultados constituyen un punto de partida para discutir las elecciones tecnológicas de estos grupos en relación con las preparaciones pigmentarias.

Palabras clave: mezclas pigmentarias, análisis MEB-EDX, cerámica Goya-Malabrigo, cuenca del río Paraná, Holoceno Tardío

This paper presents the initial results of the physical-chemical analysis of red and white pigment mixtures used to decorate archaeological ceramics found at sites in the middle and lower Paraná River basin (Entre Ríos province, Northeast Argentina). The material analyzed corresponds to ceramic assemblages associated with Goya-Malabrigo groups, chronologically situated at the end of the Late Holocene. The SEM-EDX technique is used to provide a preliminary characterization of the paints inorganic elements. The analysis revealed high concentrations of Fe in red paints, while white paints displayed some variability in their composition, but with notable levels of $\mathrm{Ca}$ and $P$. These results provide a starting point for a discussion of the groups technological choices in regard to pigment preparation.

Key words: pigment mixtures, SEM-EDX analysis, GoyaMalabrigo ceramics, Paraná River basin, Late Holocene

* Flavia V. Ottalagano, CONICET / Instituto Nacional de Antropología y Pensamiento Latinoamericano, 3 de febrero 1378, C1426BJN, Ciudad de Buenos Aires, Argentina, email: flaviaott7@gmail.com

* Silvia A. Domínguez, Laboratorio de Microscopia Electrónica, Gerencia Materiales, Centro Atómico Constituyentes, CNEA / Instituto Sábato, Av Gral. Paz 1499 (1650) San Martín, Buenos Aires, Argentina, email: sdoming@cnea.gov.ar

* Patricia B. Bozzano, Laboratorio de Microscopia Electrónica, Gerencia Materiales, Centro Atómico Constituyentes, CNEA / Instituto Sábato, Av Gral. Paz 1499 (1650) San Martín, Buenos Aires, Argentina, email: pbozzano@cnea.gov.ar
El empleo de técnicas fisicoquímicas para el estudio composicional de las mezclas pigmentarias utilizadas en la decoración cerámica constituye una línea de investigación que ha venido avanzando considerablemente en la arqueología argentina, especialmente en la región noroeste del país, donde la alfarería resulta un hallazgo muy abundante en los contextos arqueológicos prehispánicos (e.g., Botto et al. 1998; Zagorodny et al. 2002; Cremonte et al. 2003; Babot \& Apella 2009; Bertolino et al. 2009; De La Fuente et al. 2010; Palamarczuk \& Fernández de Rapp 2011; Acevedo et al. 2012; Bugliani et al. 2012; Marte et al. 2012; Aldazabal et al. 2014, entre otros).

En lo que respecta a la arqueología de grupos cazadores-recolectores de la cuenca Paraná-Plata, se han realizado estudios composicionales de la fracción inorgánica de pinturas rojas y blancas empleadas en la decoración cerámica (Paleo \& Pérez Meroni 2009; Frère 2015) y de la fracción orgánica de residuos de pigmentos hallados en estratigrafía (González de Bonaveri \& Frère 2002, 2004; González 2005), mediante técnicas como fluorescencia de rayos-X (FRX), difracción de rayos-X (DRX), espectrometría Raman y cromatografía gaseosa 
acoplada a espectrometría de masa. Estas investigaciones se han concentrado sobre materiales procedentes de sitios arqueológicos localizados en la depresión del río Salado y en el sector costero del río de La Plata (fig. 1), en la provincia de Buenos Aires. En relación con los tramos más septentrionales de la cuenca Paraná-Plata, se registra el estudio de engobes y pastas por medio de DRX y MEB-EDS en el sector del Paraná inferior, en la proximidad de la localidad de Baradero (provincia de Buenos Aires) (Tapia et al. 2013).

Hasta el momento, sin embargo, existe un vacío de información respecto de la composición de las mezclas pigmentarias utilizadas en la cerámica Goya-Malabrigo. Esta denominación ha sido empleada para designar una unidad arqueológica que ha tenido como rasgos distintivos la elaboración de vasijas con apéndices zoomorfos-antropomorfos, así como la manufactura de "alfarerías gruesas", también denominadas "campanas", debido a su peculiar morfología (fig. 2) (Serrano 1946, 1950; Gaspary 1950; González 1977). Las "alfarerías gruesas" suelen también estar decoradas con motivos zoomorfos y, en algunos casos, manifiestan una cubertura blanca o blanquecina en sus superficies, una característica que es compartida con las denominadas "alfarerías tubulares" del nordeste de la provincia de Buenos Aires (Vignati 1942). Como se muestra en la figura 2c, las alfarerías campaniformes constituyen artefactos cerámicos caracterizados por presentar paredes muy gruesas y dos aberturas: una basal y otra apical, motivo por el que algunos autores las han propuesto como posibles conservadores de rescoldo (Frenguelli 1927) o como sahumadores asociados a funciones rituales (Gaspary 1950). Desde la etnografía se plantea una notable carga simbólica de estas piezas, según esto las aves psitaciformes modeladas que usualmente decoran las campanas son las encargadas de distraer a los espíritus de los muertos, a fin de que no molesten a los vivos (Ottalagano \& Colobig 2010: 94).

Para explicar la recurrente cocción oxidante de las "alfarerías gruesas", que contrasta con la cocción mayormente reductora del resto del conjunto cerámico Goya-Malabrigo, se ha comentado la posibilidad de que recibieran un tratamiento especial durante su cochura, como cambios de posición o bien una cocción independiente (Ceruti 2003). Su manufactura también parece haber estado sujeta a elecciones tecnológicas particulares. La baja fluidalidad de la pasta de estas piezas, visibles a través de cortes petrográficos, sugiere que fueron manufacturadas posiblemente mediante modelado, a diferencia del resto de las alfarerías que habrían sido elaboradas fundamentalmente mediante superposición de rodetes de arcilla (Ottalagano \& Pérez
2013; Ottalagano 2013a, 2014; ver también Loponte 2008 y Pérez 2010 para alfarerías tubulares).

Entre los conjuntos cerámicos asociados a los grupos Goya-Malabrigo se encuentran asimismo recipientes decorados con pintura roja, aunque estas vasijas han sido menos enfatizadas desde el punto de vista clasificatorio. Entre las formas típicas de las vasijas monocromas se observan cuencos, escudillas y platos de contornos simples (sensu Shepard 1963; Balfet et al. 1983). El registro cerámico altamente fragmentado impide muchas veces determinar motivos y disposiciones de la decoración pintada. Sin embargo, comúnmente la decoración monocroma se desarrolla en la parte interna de los recipientes, como bandas paralelas a la línea del borde, y más raramente formando líneas quebradas (fig. 2).

La cerámica Goya-Malabrigo es considerada el estilo representativo de las sociedades que ocuparon el Paraná medio, así como parte del Paraná inferior, especialmente el delta superior y medio de este río. También este estilo es reconocible en algunos tramos del río Uruguay y costa uruguaya del río de La Plata (Ceruti 2003). En este trabajo se intenta una primera aproximación hacia el estudio de las mezclas pigmentarias rojas y blancas utilizadas en esta cerámica, por medio de la aplicación de técnicas fisicoquímicas, en particular mediante MEB-EDX (microscopia electrónica de barrido y microanálisis por dispersión de energía de rayos-X), también denominada con las siglas MEBEDS. La alfarería analizada en esta oportunidad está asociada a ocupaciones humanas cronológicamente situadas hacia el final del Holoceno Tardío, entre los $1380 \pm 100$ y los $625 \pm 46$ años AP (ver apartado siguiente), y proviene de sitios arqueológicos localizados en el tramo medio e inferior del río Paraná (provincia de Entre Ríos, Argentina) (fig. 1). La información aportada por el registro arqueológico de estos sitios sugiere su vinculación con grupos cazadores-recolectores complejos (ver discusión en Ottalagano 2013b; Ottalagano et al. 2015b). Por medio de la técnica MEB-EDX se obtiene información composicional de las pinturas, que se espera que contribuya a abordar la tecnología del color en la cuenca del Paraná, favoreciendo la discusión respecto de las potenciales materias primas empleadas en las preparaciones pigmentarias destinadas al arte cerámico.

\section{MÉTODOS Y MUESTRA}

En este trabajo se utilizó la técnica MEB-EDX para realizar el análisis fisicoquímico de las muestras. Esta técnica posibilita determinar la composición elemental de las mezclas pigmentarias y, paralelamente, facilita el 


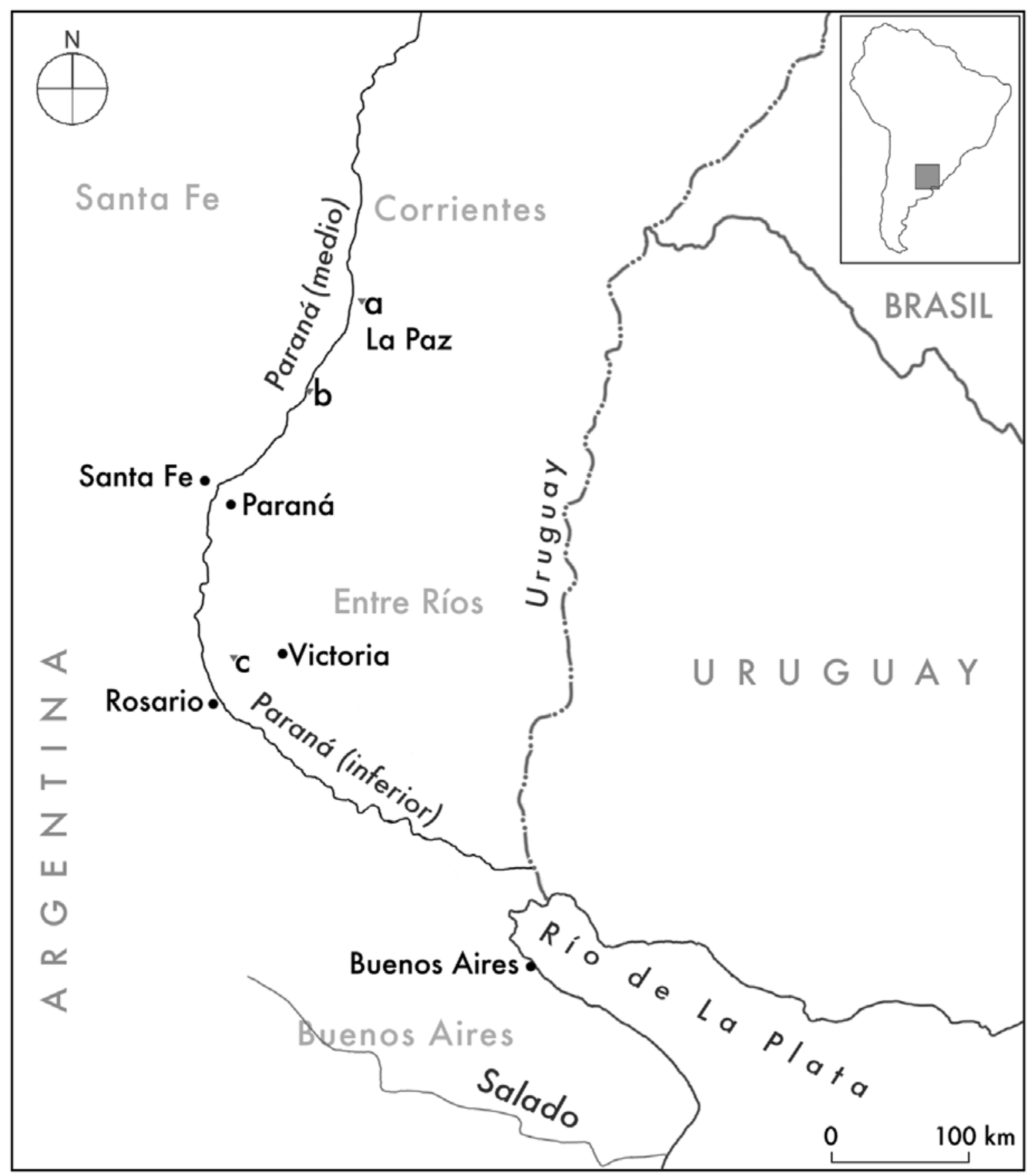

Figura 1. Área de estudio: a) sitios AAI, PCI y ELI (Paraná medio); b) sitios LPII y LPV (Paraná medio); c) sitio CPA (Paraná inferior). Figure 1. Area of study: a) AAI, PCI and ELI sites (middle Paraná); b) LPII and LPV sites (middle Paraná); c) CPA site (lower Paraná).

estudio de las superficies cerámicas a alta resolución mediante imagen. Es una técnica no destructiva que permite identificar los elementos químicos presentes en las áreas seleccionadas, y tiene una capacidad cualitativa y semicuantitativa de análisis. Si bien detecta la presencia química de carbono, no permite precisar la naturaleza de los elementos orgánicos potencialmente empleados en dichas mezclas, por ello es especialmente útil para estudiar los componentes inorgánicos de las pinturas (Garratt-Reed \& Bell 2003; Tykot 2004). La caracterización morfológica y la composición elemental de las muestras se efectuaron en los laboratorios de la 
a
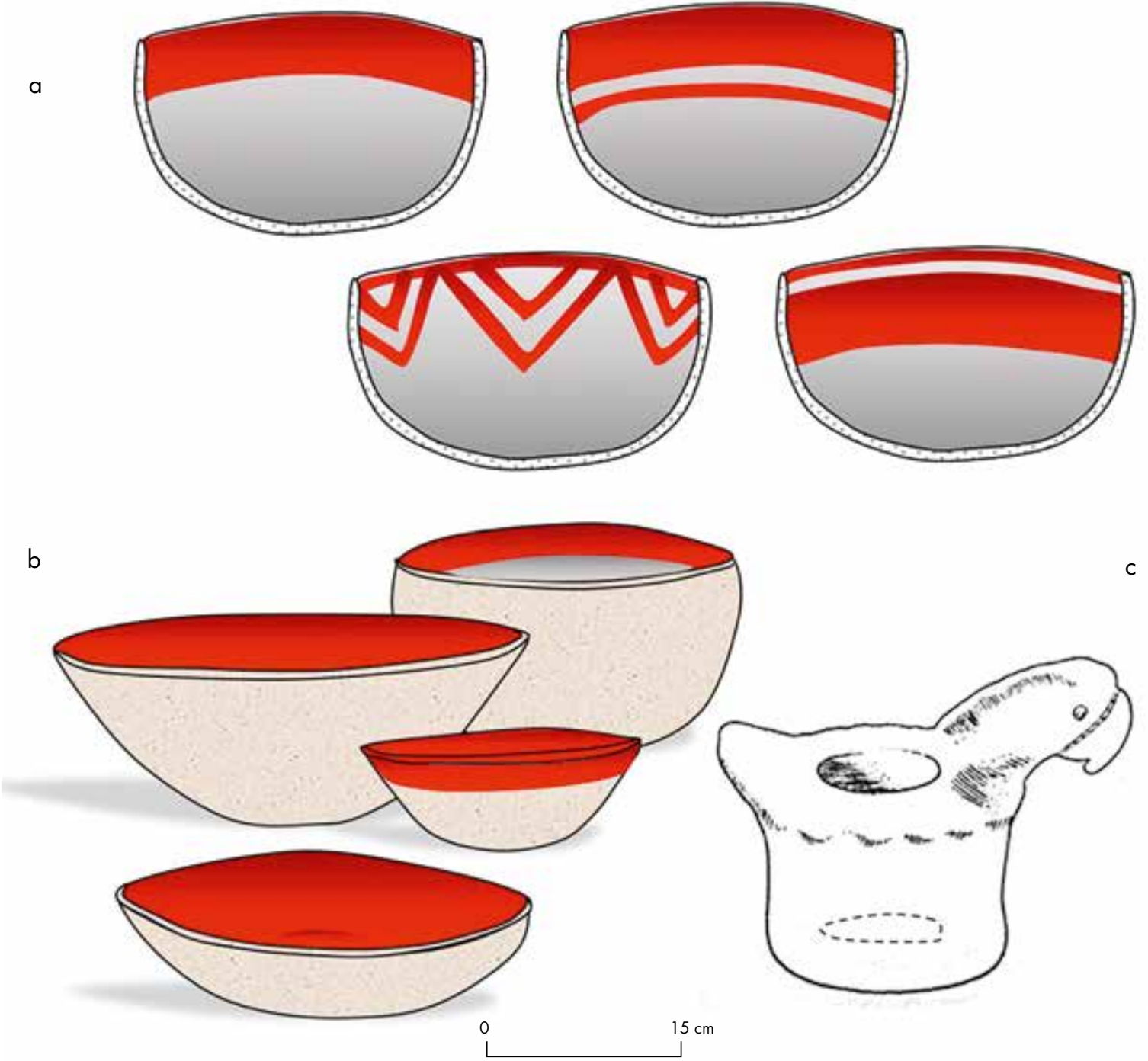

Figura 2. Esquematización de las cerámicas pintadas: a) sección interior de los recipientes (basado en Serrano 1946: 54); b) reconstrucción de principales formas; c) "alfarería gruesa" (tomado y modificado de Serrano 1946: 58).

Figure 2. Diagram of painted ceramics: a) interior cross-section of the vessels (based on Serrano 1946: 54); b) reconstruction of main forms; c) "alfarería gruesa" ("beavy pottery," adapted from Serrano 1946: 58).

Gerencia Materiales del Centro Atómico Constituyentes, Comisión Nacional de Energía Atómica (CNEA). Se empleó un equipo FEI Quanta 200, provisto con equipo de microanálisis dispersivo en energías de rayos-X, EDAX Genesis Apex 2. Este sistema de microanálisis detecta los elementos químicos presentes en un volumen de interacción de un micrón cúbico, por lo que es una técnica analítica adecuada para el estudio de capas de pintura debido a su escaso poder de penetración (Yacobaccio et al. 2008).

Una pintura generalmente puede definirse como una mezcla pigmentaria preparada comúnmente a base de dos o tres componentes: el pigmento o la materia prima colorante propiamente dicha y las sustancias utilizadas como aglutinante o carga. Estas últimas pueden ser tanto coloras o incoloras y tendrían por función facilitar la adhesión de las preparaciones pigmentarias a las superficies a decorar e incrementar el volumen de pintura obtenida, entre otras cuestiones (Petit \& Valot 1991). Algunos autores emplean el término pigmento, no obstante, para referirse a las sustancias o a la mezcla de sustancias que en su conjunto modifican el color de las superficies cerámicas, y que pueden ser aplicadas antes o después de la cochura de las piezas, reservando 
el término colorante para hacer alusión a los elementos de los cuales se deriva el color del pigmento (Rye 1981). También se ha propuesto utilizar la denominación de cobertura para hacer referencia a aquellos acabados de superficie que exhiben color, pero que muestran dificultades para ser discriminados, como engobe o pintura (Frère 2015). De hecho, algunas variantes de la técnica de engobe implican el agregado de algún material colorante al fluido arcilloso que lo conforma (Shepard 1963; Rye 1981; Caruso 1985; García Rosselló \& Calvo Trias 2013), en cuyo caso la línea de separación entre estos engobes y las pinturas precocción se vuelve sutil. Este trabajo enfatizó como objeto de estudio las mezclas pigmentarias aplicadas a la cerámica, ya sea para pintar una sección particular de la pieza o bien para aportar color a la totalidad de su superficie.

La alfarería analizada procede de seis sitios multifunción, ubicados en diferentes tramos de la cuenca media e inferior del río Paraná (provincia de Entre Ríos, nordeste de Argentina) (fig. 1), y son los siguientes: La Palmera II y V (LPII y LPV), Puerto Cuartel I (PCI), Arroyo Arenal I (AAI), Arroyo El Largo I (ELI) y Cerro Puesto Acosta (CPA). Se seleccionaron 11 tiestos de cerámica con rastros de pinturas precocción rojas y blancas, halladas en estratigrafía y en contextos domésticos (Ceruti 1984, 1989, 2003; Ottalagano et al. 2010, Ottalagano et al. 2015b). En estos sitios, la cerámica con decoración a base de pintura roja representa en promedio 15\% del total de bordes cuantificados (LPII: $\mathrm{n}=497$; PCI: $\mathrm{n}=$ 138; AAI: $\mathrm{n}=608$; ELI: $\mathrm{n}=300$; CPA: $\mathrm{n}=354$ ), mientras que el porcentaje de tiestos que exhibe una cubertura blanca es aún menor, aproximándose a $2 \%$.

Las muestras estudiadas se vinculan con los siguientes fechados, realizados sobre muestras de carbón,

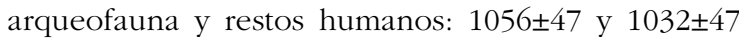
años AP (sitio LPII) (Ottalagano et al. 2015b), 1380 \pm 100 y 900 \pm 120 (sitio ELI) (Ceruti 2003). También se dispone de una nueva datación para el sitio AAI, de 625 446 años AP (AA102684), la que ha sido efectuada sobre restos humanos mediante técnica AMS. Estas cronologías son congruentes con la informada para otros sitios con alfarería Goya-Malabrigo del delta superior, también localizados en la provincia de Entre Ríos (ver Bonomo et al. 2011).

Se seleccionaron fragmentos pintados en dos tonalidades de rojos, los cuales son los más usuales de encontrar en el registro arqueológico considerado: rojo (ca. 5R 4/10, 4/12) (muestras CPA-1, AAI-1, ELI-1, LPV-1, PCI-1) y rojo castaño (ca. 2.5YR 4/6) (muestras LPII-2, AAI-2, ELI-2). La decoración a base de pintura roja está situada en el interior de las vasijas, y en las muestras ELI-1, LPII-2 y AAI-2 fue posible identificarla como pintura zonal, dispuesta como anchas franjas paralelas a la línea del borde. En el caso de las cuberturas blancas, los tonos no son puros, sino más bien tienden a ser blanquecinos ( $c$ a. 7.5YR 8/1, 8/2). Las tres muestras con cubertura blanca corresponden a "alfarerías gruesas".

Algunas muestras han sido analizadas petrográficamente en el marco de trabajos previos (LPII-1, LPII-2, LPII-3 y CPA-2), lo que ha permitido identificar pastas con tiesto molido intencionalmente agregado y con la presencia de inclusiones minerales naturalmente presentes en los bancos sedimentarios locales, como granos félsicos (cuarzos, feldespatos y plagioclasas) y nódulos de óxidos férricos (Ottalagano \& Pérez 2013; Ottalagano 2013a, 2014). Las pastas de los tiestos analizados presentan diferencias cromáticas. Se registran colores oscuros en los fragmentos con pintura roja (Munsell 10 YR 4/1, 3/1, 2/1) y coloraciones claras en las muestras de "alfarería gruesa" que presentan cuberturas blancas (Munsell 5 YR 8/4, 7/6). Estas diferencias podrían estar vinculadas a cocciones cortas o a atmósferas reductoras, para el primer grupo cromático, y a atmósferas oxidantes, cocciones prolongadas o con mayores niveles de temperatura, para el segundo grupo cromático (Shepard 1963; García Rosselló \& Calvo Trias 2006, ver discusión en Ottalagano et al. 2015a).

Los fragmentos cerámicos se colocaron directamente en el portaobjetos, sin tratamiento ni preparación previa. Se analizaron pequeñas áreas cuadrangulares del orden de $\operatorname{los} 50$ a $200 \mu \mathrm{m}$ de lado. Se midieron entre una y tres microáreas para cada tiesto, debido a que los pigmentos arqueológicos pueden no resultar homogéneos (Acevedo et al. 2012). En ciertos casos se tomaron también datos composicionales de las inclusiones más abundantes presentes en la superficie coloreada (EDX 3 de las muestras CPA-1 y CPA-2, Tabla 1), cuyos tamaños oscilaban entre los 6-10 $\mu \mathrm{m}$, es decir, superiores a la fracción arcilla establecida en 0,0039 mm según la escala Udden-Wentworth (Adams et al. 1984). Asimismo, se aplicaron análisis sobre superficies cerámicas sin pigmentar y sobre el perfil de un corte fresco, con el objetivo de obtener información composicional de la matriz cerámica y poder compararla con las mediciones obtenidas en las cuberturas (Tabla 1).

\section{RESULTADOS Y DISCUSIÓN}

El análisis revela que todas las mezclas pigmentarias manifiestan una composición elemental mayormente similar, pero con variaciones en la concentración de determinados elementos. Las proporciones de hierro (Fe), calcio $(\mathrm{Ca})$ y fósforo $(\mathrm{P})$ resultan particularmente variables según la coloración de la pintura. Así, el color rojo de las 
Tabla 1. Cuantificación de elementos químicos por muestra, obtenido del análisis MEB-EDX.

Table 1. Quantification of chemical elements per sample, obtained from SEM-EDX analysis.

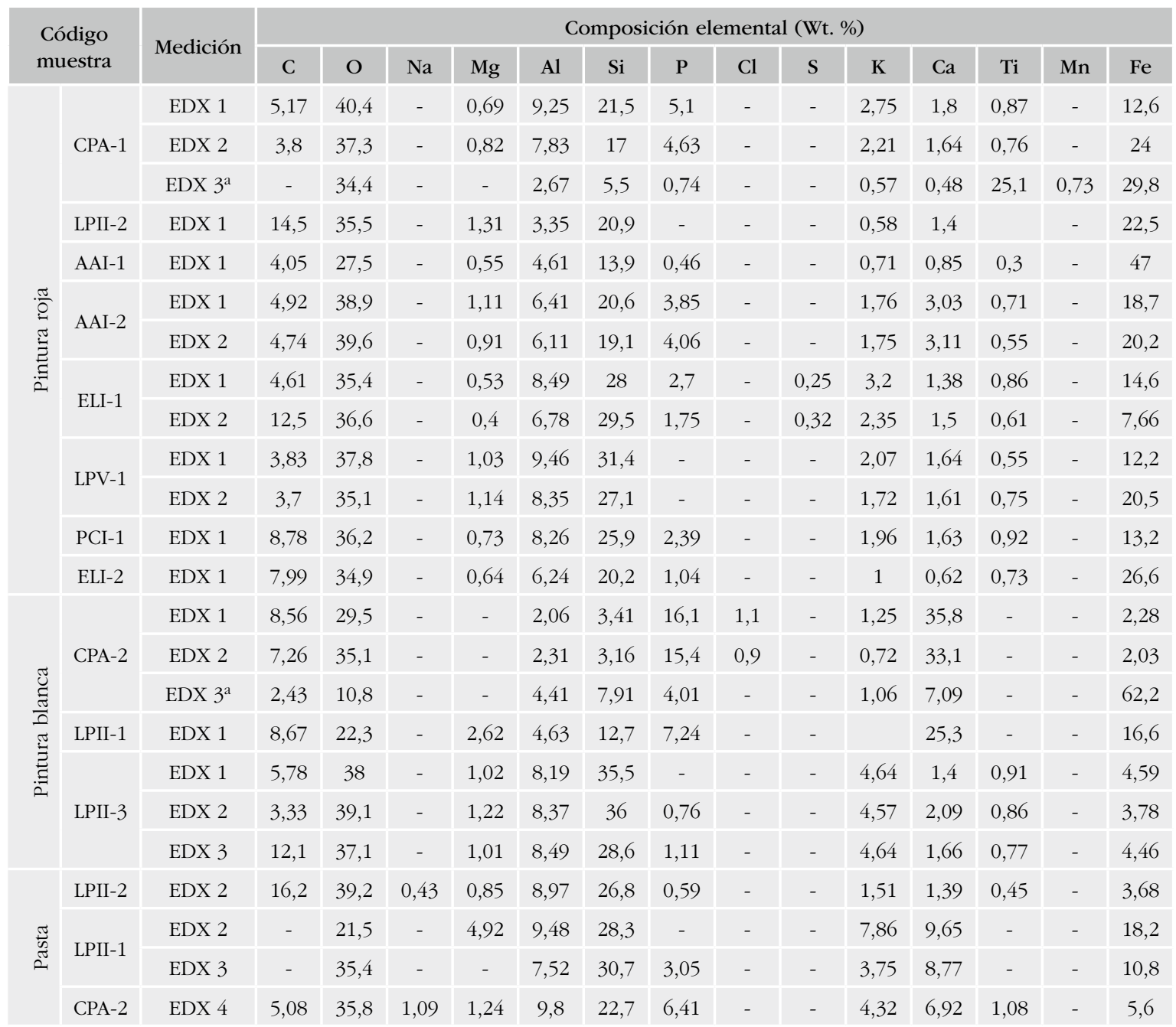

(a) Composición elemental de inclusiones presentes en las preparaciones pigmentarias que estarían asociadas con minerales de Fe.

pinturas se originaría a partir de altas concentraciones de $\mathrm{Fe}$, en tanto que las cuberturas blancas derivarían de sustancias ricas en Ca y P (Tabla 1, Gráfico 1).

$\mathrm{El} \mathrm{Fe}$ es un elemento que se encuentra también presente en el resto de las muestras analizadas, por lo que se considera que en estos casos forma parte de la composición mineralógica de las materias primas arcillosas utilizadas. No obstante, las pinturas rojas presentan en promedio $20 \%$ de contenido de hierro en su composición elemental, esto es $15 \%$ más de Fe que aquel encontrado en promedio en la matriz cerámica y en las cuberturas blancas (Tabla 1). Si se comparan las distintas mediciones EDX realizadas en una misma muestra se advierte la existencia de ciertas variaciones en los niveles de $\mathrm{Fe}$ detectados en las diferentes microáreas analizadas de la superficie cerámica pintada de un mismo tiesto (Tabla 1). Este hecho puede estar relacionado con preparaciones pigmentarias insuficientes mezcladas al momento de ser aplicadas sobre las superficies cerámicas, aunque también esto puede estar asociado con alteraciones sufridas por las pinturas a causa del uso o bien por factores postdepositación que contribuyeran a desprender o desgastar parte de las cuberturas. Es de destacar que aquellas muestras cuyo color se identificó como rojo castaño ( $c a .2 .5 Y R$ 4/6), como es el caso de las muestras LPII-2, AAI-2 y ELI-2, 


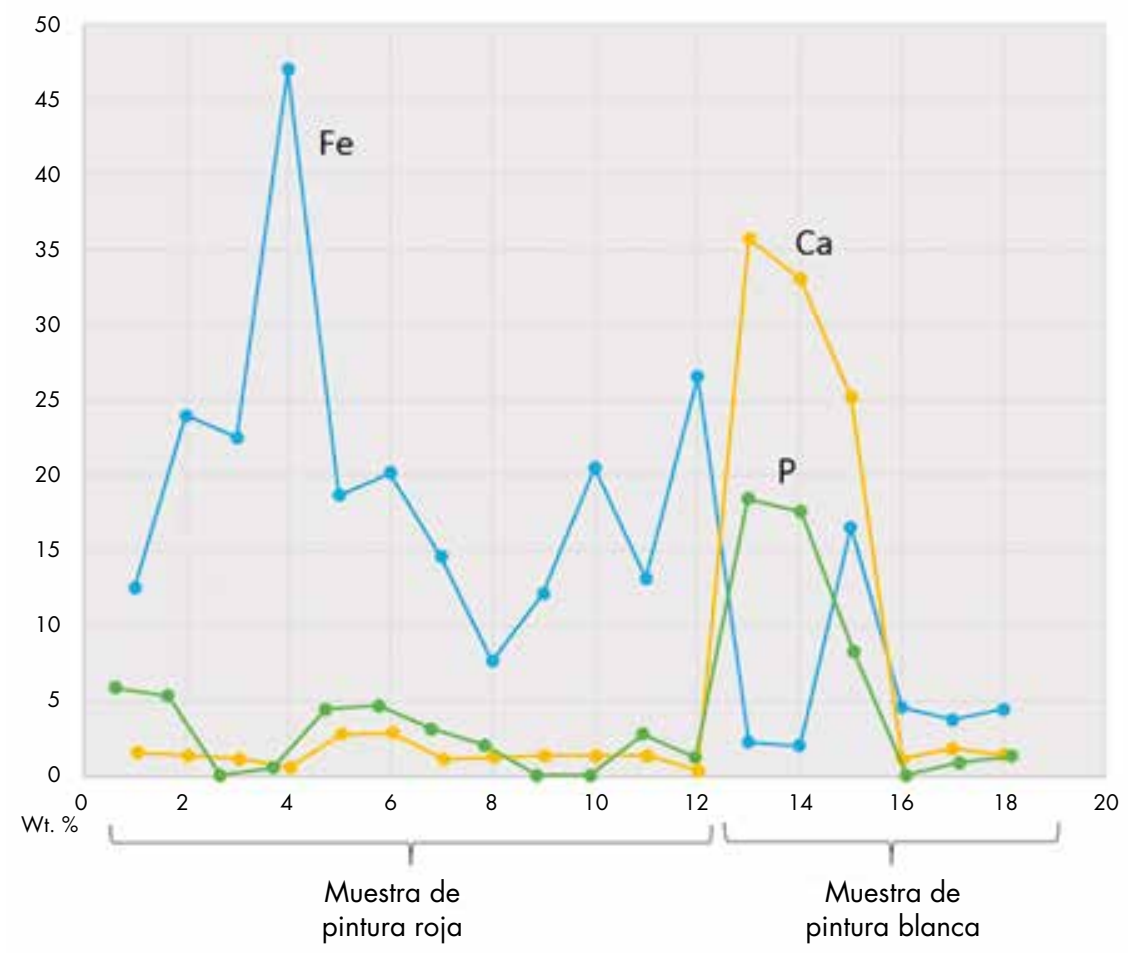

Gráfico 1. Concentración diferencial de hierro, calcio y fósforo en las muestras.

Graph 1. Differential concentration of iron, calcium and phosphorus in the samples.

no mostraron diferencias composicionales significativas respecto de las muestras agrupadas como color rojo (ca. 5R 4/10, 4/12).

El Fe podría indicar la presencia de óxidos férricos como la hematita $\left(\mathrm{Fe}_{2} \mathrm{O}_{3}\right)$, la que ha sido identificada petrográficamente como una inclusión mineral abundante en las pastas cerámicas de los sitios LPII y CPA, de los cuales ahora se posee información al respecto (Ottalagano \& Pérez 2013: 72; Ottalagano 2013a: 65, 2014), así como también pudo ser registrada en la alfarería de otros sitios arqueológicos también localizados en la cuenca del Paraná (Loponte 2008: 135; Pérez 2010: 83; Ottalagano \& Pérez 2013: 72; Tapia et al. 2013: 7). La hematita ha sido detectada como componente principal de las pinturas rojas en cerámicas provenientes de sitios arqueológicos localizados en la depresión del Salado, en la baja Cuenca del Plata (Frère 2015), así como en varios otros casos arqueológicos (e.g. Bertolino et al. 2009; De La Fuente et al. 2010; Acevedo et al. 2012; Bugliani et al. 2012; Marte et al. 2012). En la cuenca del Paraná, los grupos humanos podrían haberse abastecido localmente de este mineral. La presencia de hematita en la litología del delta del Paraná es mencionada por Cavallotto y colaboradores (2005), en tanto que Herbst
(2000), aunque no brinda detalles de su composición química, señala la recurrencia de geodas ferruginosas en los sedimentos de la Formación Ituzaingó, cuya distribución abarca amplias zonas del Paraná medio e inferior.

Las altas concentraciones de silicio $(\mathrm{Si})$ y aluminio (Al) en la cerámica pintada indican la presencia de aluminosilicatos, como es el caso de las arcillas, en las mezclas pigmentarias. De hecho, los análisis mediante EDX han permitido identificar similitudes composicionales entre la matriz cerámica y las pinturas (Tabla 1). Las arcillas, además de estar constituidas fundamentalmente por aluminosilicatos, con frecuencia contienen también cantidades apreciables de óxidos férricos y elementos minoritarios, como óxidos de titanio, álcalis (e.g., $\mathrm{K}$ y $\mathrm{Na}$ ) y elementos alcalinotérreos (e.g., Ca y Mg) (Besoain 1985; Solá 2004), los que se han cuantificado en baja proporción en casi todas las muestras de pintura. La combinación de hierro (Fe) y titanio (Ti) en la medición 3 de la muestra CPA-1 (Tabla 1), que corresponde al análisis EDX de una partícula específica, probablemente indique la presencia de ilmenita $\left(\mathrm{FeTiO}_{3}\right)$. Este es un óxido de hierro que puede contener manganeso (Mn) y magnesio $(\mathrm{Mg})$, y que frecuentemente se halla en los sedimentos, al igual que otros óxidos que poseen 
Ti (Besoain 1985). Su baja proporción en las mezclas pigmentarias, así como su identificación en la matriz cerámica, hace pensar que el Ti es también un elemento inherente a la arcilla utilizada. Estos datos sugieren que las pinturas fueron elaboradas teniendo como base una preparación arcillosa, la que habría funcionado como vehículo del material colorante propiamente dicho. La mezcla de colorantes con sustancias que tienen una función aglutinante o de carga es bien conocida (Petit \& Valot 1991). Los trabajos experimentales señalan que la dilución de sustancias colorantes en un compuesto de arcilla y agua facilita la adhesión de la preparación a las superficies cerámicas (Frère 2015), y para algunos autores esta decisión tecnológica es considerada incluso una variante de la técnica de engobe (Shepard 1963: 68; Rye 1981: 41; García Rosselló \& Calvo Trias 2013: 66).

Como ejemplo de pinturas rojas, en la figura 3 a se presenta el espectro EDX de la muestra AAI-1, donde se observa una proporción muy elevada de Fe. Esta muestra corresponde a un fragmento de borde perteneciente a un recipiente abierto con pintura interna muy bien conservada. Dentro de las cuberturas rojas, esta muestra es la que menos proporción de aluminosilicatos contiene, por lo que se estima que el material colorante se aplicó más puro, es decir, con menor proporción de arcilla como carga. En la figura 3 b se detalla el espectro EDX de la muestra PCI-1 que exhibe, por el contrario, mayor proporción de material arcilloso y relativamente menor concentración de material colorante, bajo la forma de Fe. Como se observa en la figura 4, el espesor de la capa de pintura es muy delgado, y varía entre los 14 $\mu \mathrm{m}$ y los $43 \mu \mathrm{m}$.

Las pinturas o cuberturas blancas, por su parte, mantienen mayor variación en cuanto a su composición elemental. Las muestras CPA-2 y LPII-1 destacan por presentar una significativa concentración de fósforo (P) y de calcio ( $\mathrm{Ca}$ ). Estos elementos habrían formado parte esencial de las materias primas colorantes incorporadas en las mezclas pigmentarias de ambas muestras, originando el color blanquecino (Gráfico 1, figs. 3 y 4). Particularmente se observa que la concentración de aluminosilicatos en estas dos muestras es baja. Es posible entonces que los elementos $\mathrm{P}$ y Ca hayan integrado compuestos que podrían haber actuado además como carga en las preparaciones pigmentarias, minimizando -por consiguiente- la necesidad de agregar una mayor cantidad de material arcilloso a la preparación. Cabe la alternativa, asimismo, que se hayan incorporado sustancias aglutinantes de base vegetal o animal en estas preparaciones. Sin embargo, como se dijo antes, la técnica MEB-EDX no contribuye a identificarlas.
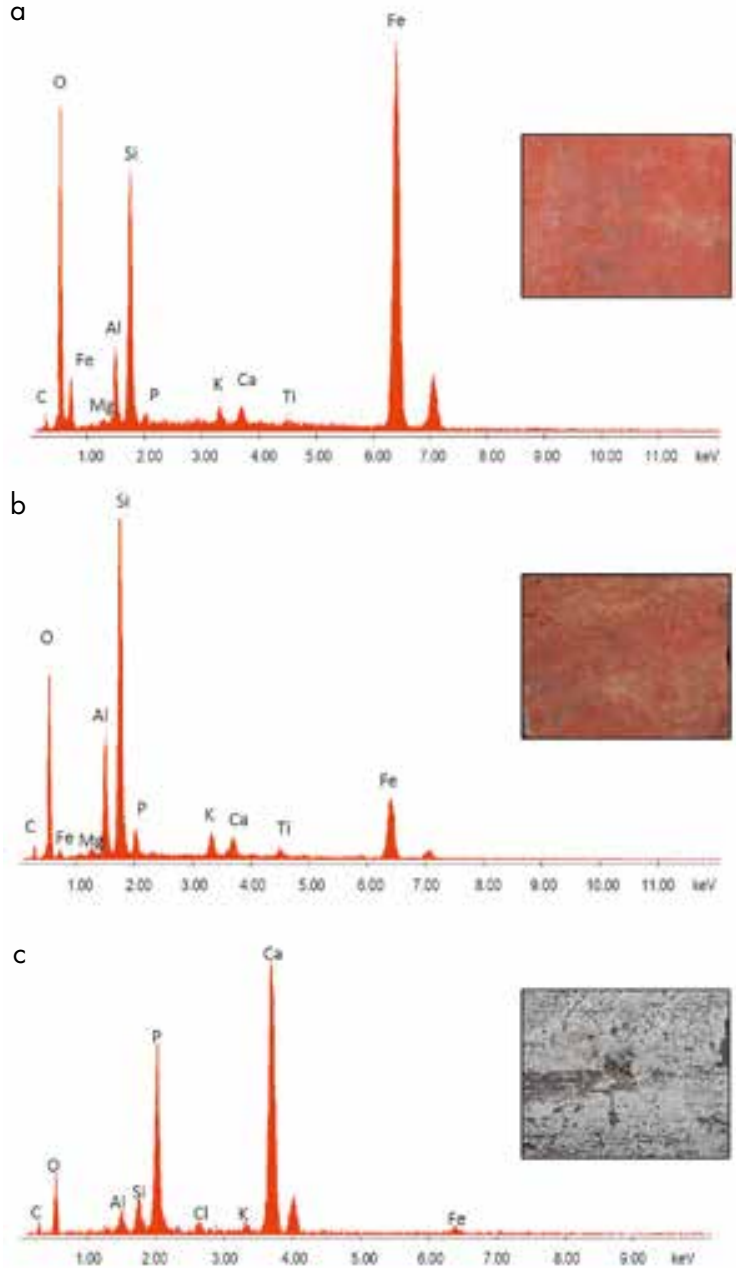

Figura 3. Espectros EDX de las muestras: a) pintura roja con alto contenido de $\mathrm{Fe}$; b) pintura roja con mayor concentración de aluminosilicatos; c) pintura blanca con elevado contenido de calcio y fósforo.

Figure 3. EDX spectra of the samples: a) red paint with high Fe content; b) red paint with bighest concentration of aluminosilicates; c) white paint with bigh calcium and phosphorus content.

La presencia de altos contenidos de calcio ( $\mathrm{Ca}$ ) en las muestras CPA-2 y LPII-1 podría derivar del empleo de carbonatos. Las valvas de moluscos son particularmente ricas en carbonato de calcio $\left(\mathrm{CaCO}_{3}\right)$ y podrían haber sido utilizadas como colorante, debido a que son recursos muy abundantes en los sitios arqueológicos del área de estudio. También se ha mencionado como hipótesis el potencial uso de cáscaras de huevo de aves en la elaboración de cuberturas blanquecinas (Frère 2015), que también son ricas en este compuesto. En muchos casos se ha reportado asimismo el empleo del yeso o sulfato de calcio dihidratado $\left(\mathrm{CaSO}_{4} \cdot 2 \mathrm{H}_{2} \mathrm{O}\right)$ en los pigmentos (e. g., Barbosa \& Gradin 1986-1987; 

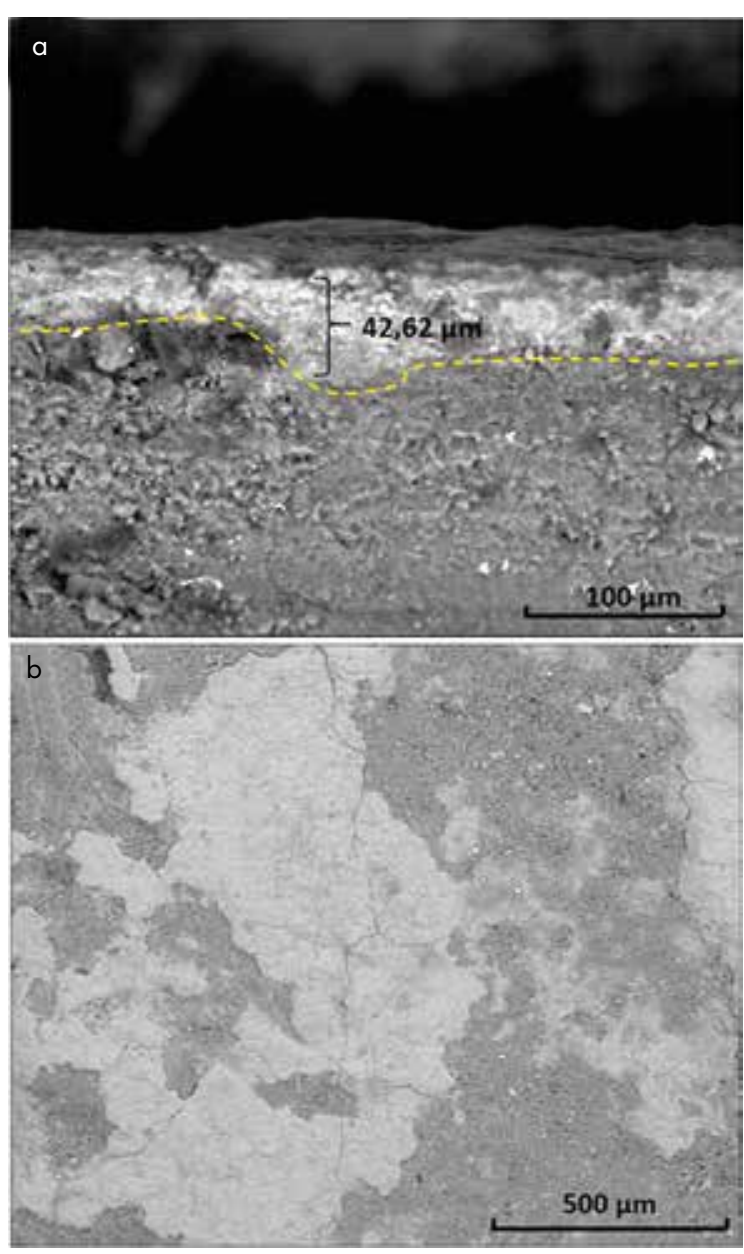

Figura 4. Fotomicrografías mediante MEB: a) pintura roja, vista en el perfil del fragmento (magnificación: 800x); b) pintura blanca distribuida irregularmente sobre la superficie del tiesto (magnificación: 180x).

Figure 4. SEM photomicrographs: a) red paint fragment in profile (800x magnification); b) white paint irregularly distributed on the ceramic surface (180x magnification).

Boschín et al. 2002; Yacobaccio et al. 2008; Palamarczuk \& Fernández de Rapp 2011; Sepúlveda et al. 2014). Esta opción sin embargo queda descartada, ya que en las pinturas blancas analizadas no se detectó azufre (S), que sí está presente en este compuesto. Este elemento se halló solamente, y en mínima proporción, en la muestra ELI-1, correspondiente a un fragmento con pintura roja (Tabla 1), posiblemente como resultado de contaminantes propios del sedimento.

La combinación de Ca y P en las muestras analizadas sugiere particularmente la presencia de hidroxiapatita $\left(\mathrm{Ca}_{5}\left(\mathrm{PO}_{4}\right) \mathrm{OH}\right)$, compuesto primordial de los huesos. Este ha podido ser reconocido como fuente de color blanco en cerámicas arqueológicas y en el arte rupestre de diversas regiones, representando el uso de huesos calcinados y pulverizados (e.g., Babot \& Apella 2009; Sepúlveda 2011; Marte et al. 2012; Frère 2015). Es de destacar que si bien en las cuberturas blancas el fósforo tiende a aparecer en concentraciones más elevadas, no es exclusivo de estas. El fósforo se encuentra también presente en las pinturas rojas y en la matriz cerámica, planteando dos alternativas complementarias: a) se emplearon sustancias ricas en $\mathrm{P}$ también en las preparaciones pigmentarias rojas, aunque en menor proporción que en aquellas blancas; b) la presencia de $\mathrm{P}$ en la matriz cerámica y en las pinturas rojas se debe a factores contaminantes. Con relación a esto último, se ha advertido la posible contaminación de los fragmentos por acumulación de minerales secundarios en los poros, por efecto del uso o del contexto postdepositación (Cremonte \& Bugliani 2006-2009). También es posible que el P se encuentre relacionado con otros minerales fosfatados, para ello se deberán sumar técnicas arqueométricas adicionales para el análisis de las muestras, que aporten hacia la identificación de los compuestos específicos incluidos en las preparaciones pigmentarias.

La muestra LPII-3, por su parte, advierte una alta proporción de aluminosilicatos, con concentraciones de Si y Al que son similares a los detallados en los espectros EDX de la matriz cerámica. Esta muestra procede del mismo sitio arqueológico que la muestra LPII-1, mencionada previamente, señalando variabilidad intrasitio de las mezclas pigmentarias. Particularmente en esta muestra, al contrario con lo sucedido en el caso de las muestras LPII-1 y CPA-2, los niveles de fósforo (P) y calcio ( $\mathrm{Ca}$ ) no son tan elevados, igualando incluso aquellos hallados en las pinturas rojas y en el fondo de pasta. Esto sugiere que, en el caso puntual de esta vasija, la cubertura blanca debió estar constituida básicamente por una dilución arcillosa, que probablemente aportó el tinte blanquecino a las superficies cerámicas.

Algunos casos arqueológicos y etnográficos mencionan pigmentos a base de arcillas blancas (e. g., Sepúlveda 2011; García Rosselló \& Calvo Trias 2013). La caolinita constituye, por ejemplo, una arcilla de partículas muy finas y de alta pureza, que sobresale entre otras muchas propiedades por su blancura, así como por su poder cubriente y adherente (Besoain 1985; Bartolomé 1997). La misma ha podido ser identificada positivamente como un ingrediente de las pinturas blancas utilizadas en el arte rupestre (Boschín et al. 2002). Asimismo, se ha verificado la mezcla de caolín con hematita para lograr un color naranja (Podestá et al. 2008).

La caolinita forma parte de los bancos de arcillas que afloran naturalmente en la cuenca media y en parte de la cuenca inferior del río Paraná, pudiendo 
estar potencialmente disponible para las poblaciones locales. Se menciona que algunos bancos de arcilla de la Formación Ituzaingó pueden contener incluso un porcentaje de más del 90\% de caolinita, y una presencia subordinada de montmorillonita e illita (Herbst 2000). La caolinita, no obstante, puede contener minerales accesorios como óxidos de hierro, manganeso y titanio, además de contaminantes orgánicos, que pueden hacerle perder blancura (Bartolomé 1997). Las propiedades de la caolinita la vuelven desventajosa para la manufactura cerámica por su baja plasticidad. Sin embargo, al poseer una gran capacidad hidrofílica que le permite disolverse óptimamente en un medio acuoso y al ser altamente adherente (Bartolomé 1997; Solá 2004; Carretero \& Pozo 2008) podría constituirse como una sustancia adecuada para funcionar como cubertura. Algunos autores han mencionado, no obstante, que las cuberturas realizadas con materias primas arcillosas diferentes a la del cuerpo cerámico pueden ocasionar potenciales craquelados en las mismas, debido a los comportamientos propios derivados de la composición de cada arcilla (García Rosselló \& Calvo Trias 2006: 315).

\section{CONSIDERACIONES FINALES}

Este trabajo constituye una primera aproximación al uso de técnicas fisicoquímicas para el análisis de las mezclas pigmentarias presentes en cerámicas monocromas Goya-Malabrigo, procedentes de sitios arqueológicos localizados en la cuenca media e inferior del río Paraná. Los primeros resultados obtenidos evidencian el manejo de distintas recetas para la elaboración de las cuberturas blancas, vinculadas a las denominadas "alfarerías gruesas". Las pinturas rojas, en tanto, tienden a presentar menor variabilidad en su composición elemental.

En el caso de las preparaciones pigmentarias rojas se reconoció una alta recurrencia de hierro ( $\mathrm{Fe}$ ), el que se habría mezclado con una proporción variable de material arcilloso, a modo de carga o aglutinante. Si bien no se dispone al momento de datos químicos que permitan clarificar de qué compuesto férrico se trata, es probable que el Fe hallado en las muestras se deba a la presencia de hematita, identificada como inclusión en las pastas cerámicas provenientes de algunos de los sitios considerados en este trabajo (Ottalagano \& Pérez 2013; Ottalagano 2013a, 2014). Gracias a su estabilidad cristaloquímica, la hematita presenta durabilidad en el transcurso del tiempo, volviéndose una materia prima idónea para preparaciones pigmentarias. Por lo general, los óxidos de hierro ofrecen buena resistencia al intemperismo y a la degradación lumínica (Solá et al. 2013: 80), y esta podría ser una de las razones, junto con su abundancia en la naturaleza, que permitirían explicar su amplia utilización prehispánica como material colorante en el arte de diferentes regiones y contextos arqueológicos (e. g., Barbosa \& Gradin 1986-1987; Boschín et al. 2002; Podestá et al. 2008; Yacobaccio et al. 2008; Bertolino et al. 2009; De La Fuente et al. 2010; Sepúlveda 2011; Acevedo et al. 2012; Bugliani et al. 2012; Marte et al. 2012; Solá et al. 2013; Aldazabal 2014; Frère 2015, entre otros).

Respecto de las pinturas blancas analizadas se distinguen aparentemente al menos dos tipos de mezclas pigmentarias. Una de ellas comprende una proporción elevada de calcio ( $\mathrm{Ca}$ ) y fósforo (P), elementos que podrían provenir de compuestos como carbonato de calcio o hidroxiapatita, ya identificados como colorantes en otros contextos arqueológicos (e.g., Babot \& Apella 2009; Sepúlveda 2011; Marte et al. 2012; Frère 2015), y cuya presencia en las muestras estudiadas deberá ser contrastada mediante técnicas arqueométricas adicionales. Estos elementos se habrían aplicado más puros, es decir, con menor proporción de sustancias arcillosas, debido a los bajos porcentajes de aluminosilicatos que presentan estas muestras. No obstante, la posibilidad que se hayan agregado también aceites vegetales o grasas animales como material ligante o de carga (Petit \& Valot 1991) es una alternativa que no puede ser descartada, para ello será necesario realizar análisis de ácidos grasos para explorar esta opción.

La otra mezcla pigmentaria que ha podido ser reconocida, en relación con la pintura blanca, presenta una composición elemental muy similar a la de la matriz cerámica. Esto sugiere que se aplicó fundamentalmente un baño arcilloso, este podría haber proporcionado color blanquecino a las superficies a partir de la potencial utilización de arcillas blancas o claras, un aspecto que también deberá ser contrastado a futuro. Esto significa que no hubo una única receta respecto de la preparación de las cuberturas blancas o blanquecinas, algo particularmente destacable teniendo en cuenta especialmente que estas distintas mezclas se identificaron en dos muestras de "alfarerías gruesas" procedentes del mismo sitio arqueológico.

Los datos suministrados en este trabajo nos aproximan hacia el conocimiento de las decisiones tecnológicas relacionadas con la aplicación de color en las superficies cerámicas por parte de los alfareros prehispánicos de la cuenca del Paraná. Las conclusiones aquí referidas, sin embargo, deberán profundizarse considerando un mayor número de muestras y sumando otras técnicas analíticas. El análisis mediante MEB-EDX ha permitido cuantificar los elementos químicos inorgánicos presentes en las pinturas, pero no facilita la identificación de 
compuestos químicos específicos, lo que se espera alcanzar con la participación de técnicas arqueométricas adicionales, como DRX y espectroscopia Raman, cuya implementación aportará nueva información respecto de las sustancias incorporadas en la preparación de las mezclas pigmentarias destinadas a la decoración cerámica.

\section{REFERENCIAS}

Acevedo, V.; M. López, E. Freire, E. Halac, G. Polla \& M. Reinoso, 2012. Estudio de pigmentos en alfarería estilo negro sobre rojo de quebrada de Humahuaca, Jujuy, Argentina. Boletín del Museo Chileno de Arte Precolombino 17 (2): 39-51.

ADAMS, A.; W. MACKENZIE \& C. GUILFORD, 1984. Atlas of sedimentary rocks under the microscope. London: Longman.

AldaZABal, V.; M. Silveira, G. Custo \& M. Ortiz, 2014. Colores al norte del lago Traful, Neuquén. Boletín del Museo Chileno de Arte Precolombino 19 (2): 95-105.

Babot, M. P. \& M. Apella, 2009. Mezclas pigmentarias y acabados de superficie cerámicos: Un caso del ámbito valliserrano de Tucumán. En Problemática de la arqueología contemporánea (tomo 2), M. Tamagnini \& A. Austral, Eds., pp. 15-161. Río Cuarto: Universidad Nacional de Río Cuarto.

Balfet, H.; M. F. Fauvet-Berthelot \& S. Monzón, 1983. Pour la normalisation de la description des poteries. Paris: Centre-National de la Recherche Scientifique.

Barbosa, C. E. \& C. J. Gradin, 1986-1987. Estudio composicional por difracción de rayos-X de los pigmentos provenientes de la excavación del Alero Cárdenas (provincia de Santa Cruz). Relaciones de la Sociedad Argentina de Antropología 17 (1): 143-171, Buenos Aires.

Bartolomé, J. F., 1997. El caolín: Composición, estructura, génesis y aplicaciones. Boletín de la Sociedad Española de Cerámica $y$ Vidrio 36 (1): 7-19, Madrid.

Bertolino, S.; V. Galván Josa, A. Carreras, A. Laguens, G. De La FuENTE \& J. Riveros, 2009. X-ray techniques applied to surface paintings of ceramic pottery pieces from Aguada Culture (Catamarca, Argentina). X-Ray Spectrometry 38, 2009 [online] pp. $95-102<$ http://onlinelibrary.wiley.com/doi/10.1002/xrs. v38:2/issuetoc> ISSN: 1097-4539 [citado 25-03-15].

Besoaín, E., 1985. Mineralogía de arcillas de suelos. San José: CIDIA-IICA.

Bonomo, M.; G. Politis \& C. Gianotti, 2011. Montículos, jerarquía social y horticultura en las sociedades indígenas del delta del río Paraná (Argentina). Latin American Antiquity 22 (3): 297-333.

Botto I. L.; V. L. Barone, M. B. Cremonte \& M. A. SÁnchez, 1998. Estudios arqueométricos de cerámicas provenientes del Noroeste Argentino. Información Tecnológica 9 (6): 79-86, La Serena.

Boschín, M. T.; A. M. Seldes, M. Maier, R. Casamiquela, R. Ledesma \& G. ABAD, 2002. Análisis de las fracciones inorgánica y orgánica de pinturas rupestres y pastas de sitios arqueológicos de la Patagonia Septentrional argentina. Zephyrus 55: 183-198, Salamanca.

Bugliani, M. F.; C. Di Lello, E. Freire, G. Polla, A. Petragalli, M. Reinoso \& E. B. Halac, 2012. Empleo de espectroscopia Raman, difracción de rayos X y microscopia electrónica para el análisis de pigmentos en cerámicas Vaquerías. Boletín del Museo Chileno de Arte Precolombino 17 (2): 65-74.

Caruso, N., 1985. Cerámica Viva. Manual de uso de la técnica de elaboración cerámica. Barcelona: Omega.

Cavallotto, J. L.; R. Violante \& F. Colombo, 2005. Evolución y cambios ambientales de la llanura costera de la cabecera del río de la Plata José. Revista de la Asociación Geológica Argentina 60 (2): 353-367, Buenos Aires.
Carretero, M. I. \& M. Pozo, 2008. Mineralogía aplicada. Madrid: Thomson.

Ceruti, C. N., 1984. Investigaciones arqueológicas en el área del complejo hidroeléctrico Paraná Medio. Síntomas 8: 20-26, Buenos Aires.

Cerutt, C. N., 1989 Ms. Las modificaciones ambientales del Pleistoceno final-Holoceno y su relación con los asentamientos humanos en el noroeste de Entre Ríos. Informe final arqueológico, PIDCONICET No $3-081800 / 88$.

Ceruti, C. N., 2003. Entidades culturales presentes en la cuenca del Paraná Medio (margen entrerriana). Mundo de Antes 3: 111-135, Tucumán.

Cremonte, M. B.; M. Baldini \& I. L. Botto, 2003. Pastas y colores. Un camino al conocimiento del estilo Portezuelo de Aguada. Intersecciones en Antropología 4: 3-16, Olavarría.

Cremonte, M. B. \& M. F. Bugliani, 2006-2009. Pasta, forma e iconografía. Estrategias para el estudio de la cerámica arqueológica. Xama 19-23: 239-262, Mendoza.

De la Fuente, G.; A. Carreras, J. M. Pérez Martínez, S. Martín \& A. Riveros, 2010. Identificación y análisis de pigmentos y pinturas en cerámicas arqueológicas Sanagasta ( $c a$. AD 900-1200) e Inka (ca. AD1480-1532) a través de MEB-EDS y microespectroscopia de Raman (Abaucán, Tinogasta, Catamarca, Argentina). En $L a$ arqueometría en Argentina y Latinoamérica, S. Bertolino, R. Cattáneo \& A. Izeta, Eds., pp. 325-331. Córdoba: Editorial de la Facultad de Filosofía y Humanidades, UNC.

Frenguel.i, J., 1927. Nuevo tipo de alfarería indígena ornitomorfa. Boletín de Educación 24: 2-11, Santa Fe.

Frère, M. M., 2015. Tecnología cerámica de los cazadores-recolectores-pescadores de la microrregión del río Salado, provincia de Buenos Aires. Tesis para optar al título de Doctor, Facultad de Filosofía y Letras, Universidad de Buenos Aires.

García Rosselló, J. \& M. Calvo Trias, 2006. Análisis de las evidencias macroscópicas de cocción en la cerámica prehistórica: Una propuesta para su estudio. Mayurqa 31: 83-112, Palma de Mallorca.

García Rosselló, J. \& M. Calvo Trias, 2013. Making Pots. El Modelado de la cerámica a mano y su potencial interpretativo. Oxford: British Archaeological Reports, International Series 2540.

GaRRATT-ReED, A. J. \& D. C. Bell, 2003. Energy-dispersive $x$-ray analysis in the electron microscope. Oxford: BIOS Scientific Publishers.

GASPARY, F., 1950. Investigaciones arqueológicas y antropológicas en un "cerrito" de la isla Los Marinos (Dpto. Victoria, Entre Ríos) situada frente a Rosario. Publicaciones del Instituto de Arqueología, Lingüística y Folclore 23: 3-66, Córdoba.

GonzÁLEz, A. R., 1977. Arte precolombino de la Argentina. Introducción a su historia cultural. Buenos Aires: Imprenta Coni.

GonzÁLEZ, M. I., 2005. Arqueología de alfareros, cazadores y pescadores pampeanos. Buenos Aires: Sociedad Argentina de Antropología.

GonZález de BonAVERI, M. I. \& M. M. Frère, 2002. Explorando algunos usos de la alfarería pampeana. En Del mar a los salitrales, diez mil años de historia pampeana en el umbral del tercer milenio, D. Mazzanti, M. Berón \& F. Oliva, Eds., pp. 31-40. Mar del Plata: Universidad de Mar del Plata.

González de Bonaveri, M. I. \& M. M. Frère, 2004. Analysis of potsherds residues and vessel use in hunter-gatherer-fisher groups (Pampean Region, Argentina). En Actas del XIVth UISPP Congress, University of Liege, Le Secrétariat du Congres, Eds., pp. 27-36, Oxford: BAR International Series 1270.

HerBSt, R., 2000. La Formación Ituzaingó (Plioceno). Estratigrafía y distribución. Serie Correlación Geológica 14, 2000 [online] $<$ http://www.insugeo.org.ar/libros/cg_14/12_la_formacion_ituzaingo.htm> ISSN 1666-9479 [citado 26-03-15].

Loponte, D. M., 2008. Arqueología del humedal del Paraná inferior: Bajios ribereños meridionales. Buenos Aires: Instituto Nacional de Antropología y Pensamiento Latinoamericano.

Marte, F.; V. ACEvedo \& N. Mastrangelo, 2012. Técnicas arqueométricas combinadas aplicadas al análisis de diseños de alfarería "tricolor" de quebrada de Humahuaca, Jujuy, Argentina. Boletín del Museo Chileno de Arte Precolombino 17 (2): 53-64. 
Ottalagano, F., 2013a. The pre-Hispanic ceramic technology of South American hunter-gatherers (Paraná River Delta, Argentina): A preliminary archaeometric study. Archaeological and Anthropological Sciences 5: 59-68, Berlín-Heidelberg.

Ottalagano, F., 2013b. Aves simbólicas, estilo e identidad en la arqueología del gran río sudamericano: Un estudio contextual del arte cerámico de las sociedades prebispánicas de la cuenca del río Paraná. Buenos Aires: Instituto Nacional de Antropología y Pensamiento Latinoamericano.

OtTalagano, F., 2014. Explorando la variabilidad petrográfica entre vasijas decoradas y lisas del sitio La Palmera 2 (Entre Ríos, Argentina): Hacia un abordaje microscópico del arte cerámico prehispánico de la cuenca del río Paraná. Revista Chilena de Antropología (en prensa).

Ottalagano, F. \& M. M. Colobig, 2010. Concepciones de aves y felinos en los relatos de un informante chaná: Entrevista a Blas Jaime. Revista de la Escuela de Antropología de la Universidad Nacional de Rosario 16: 12-24, Rosario

Ottalagano, F.; M. Darigo, K. Sulich \& L. Arelovich, 2010 Investigaciones arqueológicas en el Delta superior entrerriano: Sitio Cerro Puesto Acosta (Departamento Victoria, provincia de Entre Ríos). En Arqueología argentina en el bicentenario de la Revolución de Mayo (tomo 4), R. Bárcena \& H. Chiavazza, Eds., pp.1475-1480. Mendoza: Universidad Nacional de Cuyo.

Ottalagano, F.; C. Brancatelli, M. Darigo, L. Iannelli \& B. Pereyra, 2015a. Gestos técnicos, morfología y rastros de uso en la alfarería prehispánica del sitio La Palmera 2 (provincia de Entre Ríos). Anuario de Arqueología 7: 67-84, Rosario.

Ottalagano, F.; M. Darigo, B. Pereyra, C. Brancatelli \& L. Iannelli, 2015b. Investigaciones arqueológicas en el sitio La Palmera 2 (cuenca del Paraná medio, provincia de Entre Ríos, nordeste de Argentina). Revista de Antropología del Museo de Entre Ríos 1 (1): 55-65, Paraná.

OtTalagano, F. \& M. Pérez, 2013. Estudios petrográficos comparativos: un acercamiento regional a la tecnología cerámica del Delta del Paraná. Cuadernos del Instituto Nacional de Antropología y Pensamiento Latinoamericano, Series Especiales 1 (2): 79-94, Buenos Aires.

Palamarczuk, V. \& M. E. Fernández de Rapp, 2011. Nuevos análisis sobre el relleno blanco decorativo de la alfarería Famabalasto Negro Grabado (períodos Tardío e Incaico, Noroeste argentino). En Actas del II Congreso Latinoamericano de Arqueometría, L. Vetter, R. Vega, P. Olivera \& S. Petrick, Eds., pp. 125-136. Lima: Editorial Universitaria de la Universidad Nacional de Ingeniería.

Paleo, M. C. \& M. Pérez Meroni, 2009. Relación-forma función en un conjunto alfarero del partido de Magdalena, provincia de Buenos Aires: una aproximación metodológica. En Problemáticas de la arqueología contemporánea (tomo 2), A. Austral \& M. Tamagnini, Eds., pp. 219-226. Río Cuarto: Universidad Nacional de Río Cuarto.

Pérez, M., 2010. Tecnología de producción de la alfarería durante el Holoceno tardío en el humedal del Paraná inferior. Un estudio petrográfico. Tesis para optar al grado de Licenciada en Ciencias Antropológicas. Facultad de Filosofía y Letras, Universidad de Buenos Aires.

PetTT, J. \& H. Valot, 1991. Glossaire des peintures et vernis: des substances naturelles et des matériaux synthétiques. SaintEtienne: SFIIC-ARAAFU-IFROA

Podestá, M. M.; C. Bellelli, R. Labarca, A. Albornoz, A. Vasini \& E. Tropea, 2008. Arte rupestre en pasos cordilleranos del bosque andino patagónico (El Manso, Región de Los Lagos y provincia de Río Negro, Chile-Argentina). Magallania 36 (2): 143-153, Punta Arenas.

RYe, O., 1981. Pottery Technology. Washington, DC: Taraxacum.

SEPúlVEDA, M., 2011. Pinturas rupestres y tecnología del color en el extremo sur de Chile. Magallania 39 (1): 193-210, Punta Arenas.

Sepúlveda, M.; V. Figueroa \& J. CÁrCamo, 2014. Pigmentos y pinturas de mineral de cobre en la región de Tarapacá, norte de Chile: nuevos datos para una tecnología pigmentaria prehispánica. Estudios Atacameños 48: 23-37.

Serrano, A., 1946. Arqueología del Arroyo Las Mulas en el noroeste de Entre Ríos. Córdoba: Universidad de Córdoba Ediciones.

Serrano, A., 1950. Los primitivos habitantes de Entre Ríos. Paraná: Ministerio de Educación, Serie Historia II.

SHEPARD, A., 1963. Ceramics for the archaeologist. Washington, DC: Carnegie Institution of Washington.

Solá, P., 2004 Ms. Las arcillas y el análisis petrológico de cerámica arqueológica. Apunte para la Cátedra Ergología y Tecnología de la Carrera de Ciencias Antropológicas, Orientación Arqueología, Universidad de Buenos Aires.

Solá, S.; H. Yacobaccio, M. Rosenbusch, M. S. Alonso, M. Maier, C. VÁzQuez \& M. P. CATÁ, 2013. Hematita vs. arcillas: su potencial como pigmentos rojos y su uso en tres sitios de la puna jujeña (Argentina). Boletín del Museo Chileno de Arte Precolombino 18 (1): 67-83.

TAPIA, A.; P. Solá \& M. Rosenbusch, 2013. Efectos posdepositacionales en la cerámica del sitio Rancho José, Baradero, provincia de Buenos Aires. En Arqueometría argentina. Estudios pluridisciplinarios, M. Ramos, M. Lanza, V. Helfer, V. Pernicone, F. Bognanni, C. Landa, V. Aldazabal \& M. Fernández, Eds., pp. 1-14. Luján: Editorial Aspha.

Түкот, R., 2004. Scientific methods and applications to archaeological provenance studies. En Proceedings of the international school of physics "Enrico Fermi", course CLIV, M. Martini, M. Milazzo \& M. Piacentini, Eds., pp. 407-432. Amsterdam: IOS Press.

Vignati, M. A., 1942. Alfarerías tubulares de la región de Punta Lara. Relaciones de la Sociedad Argentina de Antropología 3: 89-98, Buenos Aires.

Yacobaccio, H.; M. P. Catá, P. Solá \& M. S. Alonso, 2008. Estudio arqueológico y fisicoquímico de pinturas rupestres en Hornillos 2 (Puna de Jujuy). Estudios Atacameños 36: 5-28.

Zagorodny, N.; B. Balesta, P. Zalba \& M. Morosi, 2002. La confección de pigmentos en la producción de cerámica arqueológica (La Aguada, Catamarca, Argentina). Relaciones de la Sociedad Argentina de Antropología 27: 177-192, Buenos Aires. 